\title{
DNA import into plant mitochondria: studying of the translocation pathways in organello and in vivo
}

\author{
Tarasenko V., Tarasenko T., Klimenko E., Koulintchenko M., Subota I., \\ Shmakov V., Konstantinov Yu.* \\ Siberian Institute of Plant Physiology and Biochemistry, SB RAS, Irkutsk, Russia \\ *e-mail:yukon@sifibr.irk.ru
}

The active participation of plant mitochondria in horizontal gene transfer, which has recently received more and more evidence, one can consider as an important fundamental feature of the mitochondrial genetic system. We assume that these processes involving mitochondria are possible, as well, due to a phenomenon of the plant mitochondria natural competence to uptake ("import") DNA. Although the mechanism of DNA import into plant mitochondria is obviously of ancient evolutionary origin, it is still insufficiently studied due to the obvious complexity of mechanisms providing DNA transfer through two membranes. The working model of DNA import into the plant mitochondria includes VDAC and a precursor protein of $\beta$-subunit of ATP synthase localized in the outer membrane, CuBPp subunit of complex I in intermembrane space, and ADP/ATP carrier in the inner membrane. The results obtained to date suggest the existence of more than one mechanism of mitochondrial DNA import. To study the features of DNA import into plant mitochondria under conditions close to in vivo, we have developed and tested a new approach based on use in parallel of an (i) in organello and (ii) isolated protoplast system. In an advanced in organello system, the detection of imported DNA in the mitochondrial matrix is based on the use of fluorescently labeled substrates and on the assessment by quantitative PCR. The in vivo system is used to investigate the DNA transport from the cytoplasm to the mitochondrial matrix in Arabidopsis protoplasts. This approach allows to establish whether the regularities of the mitochondrial DNA import detected in organello are preserved in vivo. We demonstrated that DNA of various lengths is actively imported into mitochondria of Arabidopsis protoplasts. We showed that mitochondria lacking one porin isoforms, VDAC1, VDAC2 or VDAC4, transport DNA more efficiently compared to the wild-type mitochondria.

Acknowledgements: This work was financially supported by the Russian Fund for Basic Research (Grant 18-04-00603). 